\title{
Mathematical Epidemiology Model Analysis on the Dynamics of COVID-19 Pandemic
}

\author{
Abayneh Fentie Bezabih, Geremew Kenassa Edessa, Purnachandra Rao Koya
}

Department of Mathematics, Wollega University, Nekemte, Ethiopia

Email address:

abaynehf@hu.edu.et (A. F. Bezabih),gbonsa.kena@gmail.com (G. K. Edessa),drkpraophd@gmail.com (P. R. Koya)

\section{To cite this article:}

Abayneh Fentie Bezabih, Geremew Kenassa Edessa, Purnachandra Rao Koya. Mathematical Epidemiology Model Analysis on the Dynamics of COVID-19 Pandemic. American Journal of Applied Mathematics. Vol. 8, No. 5, 2020, pp. 247-256. doi: 10.11648/j.ajam.20200805.12

Received: June 2, 2020; Accepted: June 28, 2020; Published: September 8, 2020

\begin{abstract}
In the present work, Susceptible-Exposed-Infected-Recovered-Susceptible (SEIRS) mathematical model for COVID-19 Pandemic is formulated and analyzed. The positivity, boundedness, and existence of the solutions of the model are proved. The Disease-free equilibrium point and endemic equilibrium points are identified. Local Stability of diseasefree Equilibrium point is checked with the help of Next generation matrix. Global stability of endemic equilibrium point is proved using the Concept of Liapunove function. The basic reproduction number for Novel Corona virus pandemic is computed as $\mathrm{R}_{0}=(\alpha \beta \Lambda) /[(\delta+\mu)(\beta+\delta+\mu)(\gamma+\delta+\mu)]$ which depend on six different parameters. It is observed that if basic reproduction number is less than one, then number of cases decrease over time and eventually the disease dies out, and if the basic reproduction number is equals to one, then number of cases are stable. On the other hand, if the basic reproduction number is greater than one, then the number of cases increase over time gets worth. Sensitivity analysis of the basic reproduction number is done with respect to each parameter. It is observed that only some parameters $\Lambda$, $\alpha, \beta$ have high impact on the basic reproduction number. Consequently, with real data on the parameter it is helpful to predict the disease persistence or decline in the present situation. Lastly, numerical simulations are given using DEDiscover software to illustrate analytical results.
\end{abstract}

Keywords: COVID-19 Pandemic, Stability Analysis, Next Generation Matrix, Basic Reproduction Number, Simulation

\section{Introduction}

Corona virus disease 2019 (COVID-19) is an infectious disease that can cause illnesses range from the common cold to much more severe illnesses like SARS, MERS, and COVID-19 [1-3].

Severe acute respiratory syndrome corona virus 2 (SARSCov-2), commonly known as Novel Corona virus (nCoV), is a single, positive-stranded, RNA virus that belongs to Nidoviral type, which are responsible for the Current covid19 Pandemic [4]. A species that host corona virus is known to be bat. Recent research shows that SARS-CoV-2 virus and bat corona virus have $96 \%$ identical genetic sequences $[1,2]$.

The novel corona virus (nCoV) or COVID-19 may show signs of fever, cough, breathing difficulties, organ failures or even death of whole society. $[1,5]$. It can be transmitted from person to person even before any actual signs appeared, which is difficult to prevent and control [5]. Researchers all round the world have been trying to know how the virus spreads and find out effective ways control the outbreak. Compared the reproduction number $\mathrm{R}_{0}$ of severe acute respiratory syndrome of H1N1 virus (1.25), SARS (2.2-3.6), the $\mathrm{R}_{0}$ of COVID-19 indicates awful potential transmission as $2.2,3.8$ and 2.68 by different researcher in the world. WHO published an estimated $\mathrm{R}_{0}$ of COVIDh-19 is (1.4-2.5). The larger basic reproduction number $\mathrm{R}_{0}$ the greater power of transmission rate of disease and the smaller the basic reproduction number $\mathrm{R}_{0}$ the lower the transmission rate of the disease $[1,4,6]$

According to WHO report, the virus that causes COVID19 is mainly transmitted through droplets generated when an infected person coughs, sneezes, or speaks. These droplets are too heavy to hang in the air. They quickly fall on floors or surfaces. You can be infected by breathing in the virus if you are within 1 meter of a person who has COVID-19, or by touching a contaminated surface and then touching your 
eyes, nose or mouth before washing your hands. There is no specific medicine to prevent or treat corona virus disease (COVID-19). People may need supportive care to help them breathe. If you have mild symptoms, stay at home until you have recovered. You can relieve your symptoms if you:(i) rest and sleep, (ii) keep warm, (iii) drink plenty of liquids, and (iv) use a room humidifier or take a hot shower to help ease a sore throat and cough. People with COVID-19 develop signs and symptoms, including mild respiratory symptoms and fever, on an average of 5-6 days after infection (mean incubation period 5-6 days, range 1-14 days).

According to WHO report, most people infected with the COVID-19 virus will experience mild to moderate respiratory illness and recover without requiring special treatment. Older people, and those with underlying medical problems like cardiovascular disease, diabetes, chronic respiratory disease, and cancer are more likely to develop serious illness.

Novel Corona virus (2019-nCoV), Situation Report-1, Initially the Disease spread in china, republic of Korea, and Thailand and It was 282 number of confirmed cases reported globally up to 21 January 2020. Coronavirus disease 2019 (COVID-19) Situation Report-94, shows that there are 2, 695,418 peoples are infected and 188, 804 peoples are died and 739,871 peoples are recovered from Corona virus disease (Covid-19) pandemic up to 23 April 2020. Therefore it is urgent to study and provide more scientific information for a better understanding of the novel corona virus ( $\mathrm{nCoV}$ ) or Covid-19. Thus susceptible-Exposed-infectious-recoveredsusceptible (SEIRS) model is adopted to estimate the dynamics and the potential spread based on the current data of cases, to calculate the basic reproduction number $R_{0}$ under different scenarios of the epidemics and to draw preliminary conclusions about the effectiveness of public health measures like hygiene, Masks.

The main purpose of this article is to formulate and to made Mathematical model analysis that describes the disease transmission dynamics of COVID-19 based on different literature reviews. The paper will create better understanding of the current corona virus pandemic. In [2, 4] SEIR epidemic model of a data-driven analysis is done, and some parametric estimation is computed based on curve fitting and numerical methods. In [2] SIRS model of COVID-19 is constructed in the case of Indian country.

This paper is organized as follows: In section 2, Mathematical model formulation Model assumptions, description of variables and parameters, Model diagram and Model equations are presented. In section 3, Mathematical Analysis of Model: positivity, Boundedness, and existence of solution, Equilibrium points are Discussed. In section 4, Stability Analysis of Equilibrium points; Next Generation matrix, Local Stability of disease free equilibrium point (LSDFEP), Global Stability of endemic equilibrium point (GSEEP), Basic Reproduction number will be presented. In Section 5, Simulation Study of our model equations are performed with initial conditions given for the variables and some values are assigned for the parameters. The results and discussion are given in section 6. In Section 7, Conclusions and Recommendations are drawn depend on the stability analysis and simulation study.

\section{Mathematical Model Formulation}

In the present study SEIRS model of COVID-19 is Constructed as follows. The total populations are divided into four classes: (i) Susceptible class denoted by $S$ Contains population which are capable of becoming infected (ii) Exposed class denoted by $E$ consists of populations being infected but not infectious and waiting for a short period time is called latency period.(iii) Infected class denoted by I consists of population which are infected with COVID-19 and are also infectious and (iv) Recovered class denoted by $R$ consists of recovered class from infectious disease COVID-19. Mathematical SEIRS model of COVID-19 is formulated based on the following hypotheses so as to predict the past or future dynamics of COVID-19 progression and transmission Dynamics in the world.

(i) The size of total population is assumed to be constant, $N=S(t)+E(t)+I(t)+R(t)$

(ii) Both the number of births and death are may not be equal and populations are well mixed.

(iii) Susceptible class are recruited into the compartment $S(t)$ at a constant rate $\Lambda$

(iv) The Exposed class has short incubation period and not yet infective but moved to infective class at rate $\beta$

(v) Susceptible class are infected when they come into contact with COVID-19 patient and the disease transmitted according to bilinear interaction rate $\alpha \lambda(t) S$ where, $\lambda(t)=I(t)$ which is force of infection.

(vi) Recovered class revert to the susceptible class after losing their immunity at a rate $\rho$

(vii) All types of population suffer natural mortality at a rate $\mu$.

(viii) All types of population suffer die due to Covid-19 Pandemic at a rate $\delta$

(ix) All parameters in the model are positive.

Table 1. Notations and description of model variables.

\begin{tabular}{ll}
\hline Variable & Description \\
\hline $\mathrm{S}(\mathrm{t})$ & Number of susceptible individuals at timet \\
$E(t)$ & Number of Exposed individuals at time $t$ (infected but not infectious) \\
$\mathrm{I}(\mathrm{t})$ & Number of infective individuals at time $t$ (infectious class) \\
$\mathrm{R}(\mathrm{t})$ & Number of recovered individuals at time $t$ (removed, or immune) \\
\hline
\end{tabular}


Table 2. Notations and description of model parameters.

\begin{tabular}{ll}
\hline Parameter & Description \\
\hline$\Lambda$ & Constant influx of new susceptible. With this rate new susceptible class will Recruited and will enter into susceptible class \\
$\alpha$ & Infection rate or Contact rate or effective contact rate. With this rate covid-19 transfer from compartment $S$ to $E$ \\
$\beta$ & Latency transfer rate. With this rate exposed class moves from compartment $E$ to compartment $I$. \\
$\gamma$ & Recovery rate or removal rate. With this rate infected class moves from compartment $I$ to $R$ \\
$\rho$ & Loss immunity (re-infection rate). With this rate recovered class moves from compartment $R$ to $S$ \\
$\delta$ & Death rate due to infection of $C O V I D-19$. With this rate all class of compartment suffer due to the diseases. \\
$\mu$ & Natural death rate. With this rate all class of Compartment suffer natural death rate. \\
\hline
\end{tabular}

Having the above assumptions, variables, and parameters the model diagram can be given as in Figure 1

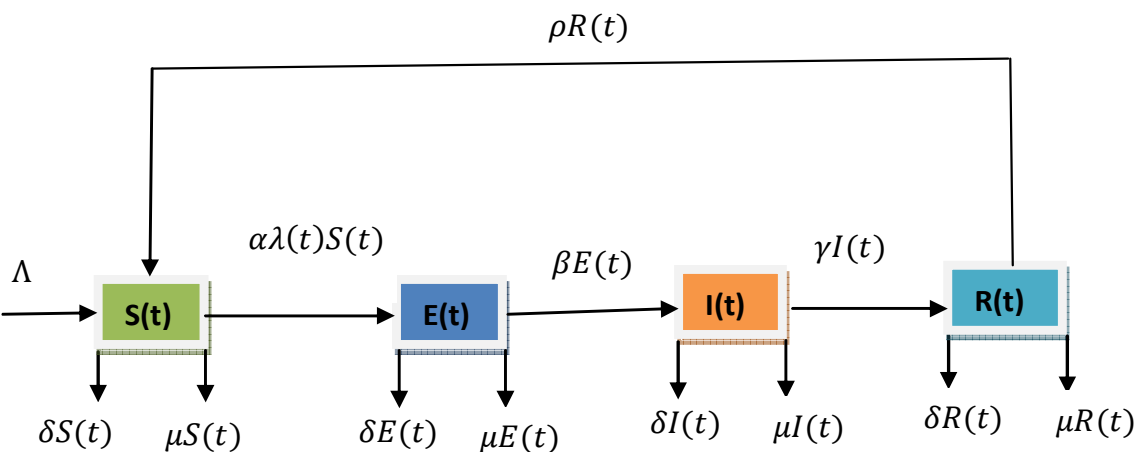

Figure 1. Model diagram.

Based on the assumptions, the notations of variables, parameters, and Model diagram, system of ordinary differential equations are formulated as follows:

$$
\begin{gathered}
d S / d t=\Lambda+\rho R(t)-\alpha S(t) I(t)-\delta S(t)-\mu S(t) \\
d E / d t=\alpha S(t) I(t)-\beta E(t)-\delta E(t)-\mu E(t) \\
d I / d t=\beta E(t)-\gamma I(t)-\delta I(t)-\mu I(t) \\
d R / d t=\gamma I(t)-\rho R(t)-\delta R(t)-\mu R(t)
\end{gathered}
$$

with initial conditions,

$S(0)>0, E(0) \geq 0, I(0) \geq 0, R(0) \geq 0, \lambda(t)=I(t)$ which is force of infection

\section{Mathematical Model Analysis}

In this section mathematical model analysis part is Presented. The analysis consists of the following features:(i) positivity of solutions the model, (ii) Boundedness of solutions of the model, (iii) Existence of solutions of the model, (iv) equilibrium points of the model: Disease free equilibrium points, endemic equilibrium points (v) Basic reproduction number (vi) Stability analysis of equilibrium points: Local stability of disease free equilibrium point and (vii) Global stability of endemic equilibrium point

\subsection{Positivity, Boundedness, and Existence of Solution}

In order to show that the model is biologically valid, it is required to prove that the solutions of the system of ordinary differential equations (1)-(4) are positive and bounded for all time [7].

Theorem 1 (Positivity) Solutions of the model equations (1)-(4) together with the initial conditions,
$S(0)>0, E(0) \geq 0, I(0) \geq 0, R(0) \geq 0 \quad$ are always positive That is, the model variables $S(t), E(t), I(t)$, and $R(t)$ are positive for all $t$ and will remain in $\mathbb{R}_{+}^{4}$.

Proof: Positivity of the model variables is shown separately for each of the model variables,

$S(t), E(t), I(t)$, and $R(t)$.

Positivity of $S(t)$ : The model equation (1) given by $d S / d t=\Lambda+\rho R-\alpha S I-\delta S-\mu S$ can be expressed without loss of generality, after eliminating the positive terms $(\Lambda+\rho R)$ which are appearing on the right hand side, as an inequality as $d S / d t \geq-(\alpha I+\delta+\mu) S$. Using variables separable method and on applying integration, the solution of the foregoing differentially inequality can be obtained as $S(t) \geq e^{-(\alpha I+\delta+\mu) t}$. Recall that an exponential function is always non-negative irrespective of the sign of the exponent, Hence, it can be concluded that $S(t) \geq 0$.

Positivity $E(t)$ : The model equation (2) arranged as $d I / d t=\alpha S I-\beta E-\delta E-\mu E$ can be expressed without loss of generality, after eliminating the positive term $(\alpha S I)$ which are appearing on the right hand side, as an inequality as $d E / d t \geq-(\beta+\delta+\mu) E$. Using variables separable method and on applying integration, the solution of the foregoing differentially inequality can be obtained as $E(t) \geq$ $e^{-(\beta+\delta+\mu) t}$. Recall that an exponential function is always non-negative irrespective of the sign of the exponent. Hence, it can be concluded that $E(t) \geq 0$.

Positivity of $I(t)$ : The model equation (3) arranged $d I / d t=\beta E-\gamma I-\delta I-\mu I$ can be expressed without loss of generality, after eliminating the positive term $(\beta E)$ which are appearing on the right hand side, as an inequality as $d I / d t \geq-(\gamma+\delta+\mu) I$. Using variables separable method and on applying integration, the solution of the foregoing differentially inequality can be obtained as $I(t) \geq$ $e^{-(\gamma+\delta+\mu) t}$. Recall that an exponential function is always 
non-negative irrespective of the sign of the exponent. Hence, it can be concluded that $I(t) \geq 0$.

Positivity of $R(t)$ : The model equation (4) arranged $d R / d t=\gamma I-\rho R-\delta R-\mu R$ can be expressed without loss of generality, after eliminating the positive term $(\gamma I)$ which are appearing on the right hand side, as an inequality as $d R / d t \geq-(\rho+\delta+\mu) R$. Using variables separable method and on applying integration, the solution of the foregoing differentially inequality can be obtained as $R(t) \geq$ $e^{-(\rho+\delta+\mu) t}$. Recall that an exponential function is always non-negative irrespective of the sign of the exponent. Hence, it can be concluded that $R(t) \geq 0$. Thus, the model variable $S(t), E(t), I(t)$, and $R(t)$ representing population size of different class are positive quantities and will remain in $\mathbb{R}_{+}^{4}$ for all $t$.

Theorem 2 (Boundedness) The positive solutions of the system of model equations (1)-(4) are bounded. That is, the model variables $S(t), E(t), I(t)$, and $R(t)$ are bounded for all $t$.

Proof: Recall that each population size is bounded if and only if the total population size is bounded. Hence, in the present case it is sufficient to prove that the total population size $N=S(t)+E(t)+I(t)+R(t)$ is bounded for all $t$. It can be shown that all feasible solutions are uniformly bounded in a proper subset $\Omega \in \mathbb{R}_{+}^{4}$ where the feasible region $\Omega$ is given by

$\Omega=\left\{(S, \quad E, \quad I, \quad R) \in \mathbb{R}_{+}^{4} ; \mathrm{N} \leq(\Lambda /(\delta+\mu))\right\} \quad$ It $\quad$ is clear that the derivative of total population with respect to time $\mathrm{t}$ is given by

$d N / d t=[d S / d t]+[d E / d t]+[d I / d t]+[d R / d t]$

Then summation of all the four model equations (1)-(4) as follows:

$$
d N / d t=[\Lambda+\rho R-\alpha S I-\delta S-\mu S]+[\alpha S I-\beta E-\delta E-\mu E]+[\beta E-\gamma I-\delta I-\mu I]+[\gamma I-\rho R-\delta R-\mu R]
$$

which simplified $\mathrm{N}(\mathrm{t}) / \mathrm{dt}=\Lambda-(\delta+\mu)(\mathrm{S}+\mathrm{E}+\mathrm{I}+\mathrm{R})$. This can be written as $\mathrm{dN}(\mathrm{t}) / \mathrm{dt}=\Lambda-(\delta+\mu) \mathrm{N}(\mathrm{t})$ Now, $\Lambda-(\delta+\mu) \mathrm{N}(\mathrm{t}) \geq 0 \quad$ if $\quad \Lambda \geq(\delta+\mu) \mathrm{N}(\mathrm{t}) \quad$ which is $\Lambda /(\delta+\mu) \geq \mathrm{N}(\mathrm{t})=\mathrm{S}+\mathrm{E}+\mathrm{I}+\mathrm{R} \geq 0$. Thus it can be concluded that $N(t)$ is bounded as it is shown that $0 \leq$ $N(t) \leq(\Lambda /(\delta+\mu))$. Therefore, $(\Lambda /(\delta+\mu))$ is an upper bound of $N(t)$. Hence, feasible solution of the system of model equations (1)-(4) remains in the region $\Omega$ which is positively invariant set. Thus, the system is biologically meaningful and mathematically well posed in the domain $\Omega$. it is sufficient to consider the dynamics of the populations represented by the model system (1)-(4) in that domain. Therefore, it can be summarized the result of theorem 2 as the model variables $S(t), E(t), I(t)$, and $R(t)$ are bounded for all $t$.

Theorem 3 (Existence) Solutions of the model equations (1)-(4) together with the initial conditions,

$S(0)>0, E(0) \geq 0, I(0) \geq 0, R(0) \geq 0$ exist in $\mathbb{R}_{+}^{4}$ i.e. the model variables $S(t), E(t), I(t)$, and $R(t)$ exist for all $t$ and will remain in $\mathbb{R}_{+}^{4}$.

Proof: Let the system of equation (1)-(4) arranged as follows: $f_{1}=\Lambda+\rho R-\alpha S I-(\delta+\mu) S$

$$
\begin{gathered}
f_{2}=\alpha S I-(\beta+\delta+\mu) E \\
f_{3}=\beta E-(\gamma+\delta+\mu) I \\
f_{4}=\gamma I-(\rho+\delta+\mu) R
\end{gathered}
$$

According to Derrick and Grossman theorem, let us now define the feasible region $\Omega$ that has been discussed under primarily results boundedness of the solutions,

$$
\Omega=\left\{(S, \quad E, \quad I, \quad R) \in \mathbb{R}_{+}^{4} ; \mathrm{N} \leq(\Lambda /(\delta+\mu))\right\}
$$

Then equations (1)-(4) have a unique solution if

$\left(\partial f_{i}\right) /\left(\partial x_{j}\right), i, j=1,2,3,4$ are continuous and bounded in $\Omega$ [7]. Here, $x_{1}=S, x_{2}=E, x_{3}=I, x_{4}=R$, The continuity and the boundedness are shown in Table 3 as follows:

Table 3. Partial derivatives of functions with respect of model variables.

\begin{tabular}{ll}
\hline For $f_{1}:$ & For $f_{3}:$ \\
$\left|\left(\partial f_{1}\right) /(\partial S)\right|=|-\alpha I-(\delta+\mu)|<\infty$ & $\left|\left(\partial f_{3}\right) /(\partial S)\right|=0<\infty$ \\
$\left|\left(\partial f_{1}\right) /(\partial E)\right|=0<\infty$ & $\left|\left(\partial f_{3}\right) /(\partial E)\right|=|\beta|<\infty$ \\
$\left|\left(\partial f_{1}\right) /(\partial I)\right|=|-\alpha S|<\infty$ & $\left|\left(\partial f_{3}\right) /(\partial I)\right|=|-(\gamma+\delta+\mu)|<\infty$ \\
$\left|\left(\partial f_{1}\right) /(\partial R)\right|=|\rho|<\infty$. & $\left|\left(\partial f_{3}\right) /(\partial R)\right|=0<\infty$ \\
For $f_{2}:$ & For $f_{4}:$ \\
$\left|\left(\partial f_{2}\right) /(\partial S)\right|=|\alpha I|<\infty$ & $\left|\left(\partial f_{4}\right) /(\partial S)\right|=0<\infty$ \\
$\left|\left(\partial f_{2}\right) /(\partial E)\right|=|-(\beta+\delta+\mu)|<\infty$ & $\left|\left(\partial f_{4}\right) /(\partial E)\right|=0<\infty$ \\
$\left|\left(\partial f_{2}\right) /(\partial I)\right|=|\alpha S|<\infty$ & $\left|\left(\partial f_{4}\right) /(\partial I)\right|=|\gamma|<\infty$ \\
$\left|\left(\partial f_{2}\right) /(\partial R)\right|=0<\infty$ & $\left|\left(\partial f_{4}\right) /(\partial R)\right|=|-(\rho+\delta+\mu)|<\infty$ \\
\hline
\end{tabular}

Thus, all the partial derivatives ,

$\left(\partial f_{i}\right) /\left(\partial x_{j}\right), i, j=1,2,3,4$ exist, continuous and bounded in $\Omega$. Hence, by Derrick and Groosman theorem, a solution for the model (1)-(4) exists and is unique.

To understand the dynamics of the system, it is necessary to identify equilibrium points of the model Equation. An equilibrium solution is a steady state solution of the model equations (1)-(4) in the sense that if the system begins at such a state, it will remain there for all times. In other words, the population sizes remain unchanged and thus the rate of change for each population vanishes. Equilibrium points of the model are found, categorized, stability analysis is done and the results have been presented in the following sub-sections.

\subsection{Disease Free Equilibrium Point}

Disease free equilibrium points are steady state solutions where there is no disease in the population. In the absence of the disease this implies that $I(t)=R(t)=0$ and the right hand side of the model is equal to zero.

Thus $\Lambda-(\delta+\mu) S=0$ which implies $S=\Lambda /(\delta+\mu)$. 
Thus, the disease-free equilibrium point of the model equation in (1)-(4) above is given by,

$$
E(S, \quad E, \quad I, \quad R)=(\Lambda /(\delta+\mu), \quad 0, \quad 0, \quad 0)
$$

\subsection{Endemic Equilibrium Point}

The endemic equilibrium point $E^{*}\left\{S^{*}, E^{*}, I^{*}, R^{*}\right\}$ in the feasible region is a steady state solution where the disease persists in the population. The endemic equilibrium point is obtained by setting rates of changes of variables with respect to time in model equations (1)-(4) to zero. That is, setting

$d S / d t=d E / d t=d E / d t=d R / d t=0 \quad$ the model equations can be written as the system of non linear equations

$$
\begin{gathered}
\Lambda+\rho R-\alpha S I-a S=0 \\
\alpha S I-b E=0 \\
\beta E-c I=0 \\
\gamma I-d R=0
\end{gathered}
$$

Where, $\quad a=\delta+\mu, b=\beta+\delta+\mu, c=\gamma+\delta+\mu, d=$ $\rho+\delta+\mu$ solving equations (7) and (8) will give expression for $I$ and $R$ in terms of variable $E$ as follows:

$$
I=(\beta / c) E
$$

$R=(\gamma / d) I=(\gamma / d)(\beta / c) E$, This expression could be re-written as

$$
R=(\beta \gamma / d c) E
$$

Now substitute (9) and (10) into (6) so as to solve $E$ which results $\alpha S(\beta / c) E-b E=0$

This can be arranged $[\alpha S(\beta / c)-b] E=0$ However, $E$ does not vanish, since the disease is assumed endemic and it is a computation of non zero equilibrium point of the system. Thus the only meaningful solution $\alpha S(\beta / c)-b=0$, then solution is given by the expression

$$
S^{*}=[b c] /[\alpha \beta]
$$

Then substituting equations (9), (10) and (11) into (5) give $\Lambda+\rho[(\beta \gamma / d c) E]-\alpha(b c / \alpha \beta)-a(b c / \alpha \beta)=0$. after some algebraic simplifications an expression for $I^{*}$ can be obtained as

$$
E^{*}=[d c\{b c(\alpha+a)-\alpha \beta \Lambda\}] /\left[\alpha \beta^{2} \gamma \rho\right]
$$

Finally, substitution of $I^{*}$ in (10) and (11) will give expressions for $I^{*}$ and $R^{*}$ in terms of parameters

$$
\begin{gathered}
I^{*}=[d\{b c(\alpha+a)-\alpha \beta \Lambda\}] /[\alpha \beta \gamma \rho] \\
R^{*}=[\{b c(\alpha+a)-\alpha \beta \Lambda\}] /[\alpha \beta \rho]
\end{gathered}
$$

Therefore the endemic equilibrium points computed above is given by

$$
\begin{gathered}
E^{*}\left(S^{*}, \quad E^{*}, \quad I^{*}, \quad R^{*}\right) \\
\left([b c] /[\alpha \beta], \quad[d c\{b c(\alpha+a)-\alpha \beta \Lambda\}] /\left[\alpha \beta^{2} \gamma \rho\right], \quad[d\{b c(\alpha+a)-\alpha \beta \Lambda\}] /[\alpha \beta \gamma \rho], \quad[\{b c(\alpha+a)-\alpha \beta \Lambda\}] /[\alpha \beta \rho]\right)
\end{gathered}
$$

\subsection{Basic Reproductive Number}

The basic reproduction number represent the average number of new infections generated by each infected person [7-9]. The higher value of $R_{o}$ the speedy the disease transmission rate and the Smaller values of $R_{o}$ the slower the disease transmission rate [12]. There are three options for the values of $R_{o}$ (i) $R_{o}<1$ means the number of new cases will decrease over time and eventually the outbreak will end on its own.(ii) $R_{o}=1$ means the cases are stable. (iii) $R_{o}>1$ means the outbreak is self-sustaining unless effective control measures are implemented. According to WHO Determining factors of basic reproduction number $R_{o}$ : (i) infectious periodhow long the infection is contagious, for instance flu typically up to 8 days and in children up to 2 weeks. The longer an infection is contagious for the higher the reproduction number.(ii) Contact rate-how many people an infected person comes into contact with $R_{o}$ will be lower if a person stays at home, higher if they are out and about. (iii) Mode of transmission (shedding potential): rapid speed transmission, if the disease transmitted by airborne, flu or measles, no physical contact or fomite necessary, and Slower transmission, the disease transmitted by body fluids, Ebola, Hepatitis B, C, or HIV. [1, 5, 9] In the discussion of disease transmission if $20 \%$ of infected individuals are responsible for $80 \%$ of transmissions those spreaders is called Super- spreaders and if $80 \%$ of infected individuals are responsible for $20 \%$ of transmissions are called mini-spreaders. [1, 5]

To drive the general reproduction number for the formulated model of Covid-19 under the discussion of primary results. $S^{*} \geq \Lambda / a$, if and only if $[b c] /[\alpha \beta] \geq \Lambda / a$.

Without losing original generality dividing both sides of the inequality by $[b c] /[\alpha \beta]$ yields $1 \geq(\alpha \beta \Lambda) /(a b c)=R_{0}$, where the letters notation a, b, c and d are given by $a=\delta+$ $\mu, b=\beta+\delta+\mu, c=\gamma+\delta+\mu, d=\rho+\delta+\mu$ and hence the basic reproduction number of the model (1)-(4) would be $R_{0}=(\alpha \beta \Lambda) /[(\delta+\mu)(\beta+\delta+\mu)(\gamma+\delta+\mu)]$.

\section{Stability Analysis}

\subsection{Stability Analysis of the Disease Free Equilibrium}

In absence of the infectious disease, the model equations have a unique disease free steady state $E_{0}$.

It is shown that DFEP of model (1)-(4) is given by $E_{0}=$ $\{[\Lambda /(\delta+\mu)], 0,0,0\}$. Now local stability analysis of DFEP is presented in the following theorem and proved with the help of next generation matrix.

Theorem 4 [Local Stability of Disease-free equilibrium points (LSDFEP)] The model equations (1)-(4) is locally asymptotically stable at disease free equilibrium point (DFEP) $E_{0}$ 
Proof: Consider the right hand side expressions of the equations (1)-(4) as functions to compute Jacobian matrix

$$
\begin{gathered}
d S / d t=\Lambda+\rho R-\alpha S I-a S \equiv f\left(\begin{array}{lll}
S, & E, \quad I, & R
\end{array}\right) \\
d E / d t=\alpha S I-b E \equiv g\left(\begin{array}{lll}
S, \quad E, \quad I, & R
\end{array}\right) \\
d I / d t=\beta E-c I \equiv h(S, \quad E, \quad I, \quad R) \\
d R / d t=\gamma I-d R \equiv k(S, \quad E, \quad I, \quad R)
\end{gathered}
$$

Where, $\quad a=\delta+\mu, b=\beta+\delta+\mu, c=\gamma+\delta+\mu, d=$ $\rho+\delta+\mu$ Now the Next generation matrix of functions $(f, g, h, k)$ with respect to $(S, E, I, R)$ is given by

$$
J\left(\begin{array}{llll}
S & E & I & R
\end{array}\right)=\left[\begin{array}{cccc}
-\alpha I-a & 0 & -\alpha I & \rho \\
\alpha I & -b & 0 & 0 \\
0 & \beta & -c & 0 \\
0 & 0 & \gamma & -d
\end{array}\right]
$$

Therefore the Next generation matrix $J$ of model at the disease free equilibrium $E_{0}$ reduces to

$$
J[(\Lambda / a) \quad 0, \quad 0, \quad 0]=\left[\begin{array}{cccc}
-a & 0 & (-\alpha \Lambda) / a & \rho \\
0 & -b & (-\alpha \Lambda) / a & 0 \\
0 & \beta & -c & 0 \\
0 & 0 & \gamma & -d
\end{array}\right]
$$

Then the eigen values of $J\left(E_{0}\right)$ are computed from characteristic equation $\operatorname{det}\left[J\left(E_{0}\right)-\lambda I\right]=0$.

$$
\left|\begin{array}{cccc}
-a-\lambda & 0 & (-\alpha \Lambda) / a & \rho \\
0 & -b-\lambda & (-\alpha \Lambda) / a & 0 \\
0 & \beta & -c-\lambda & 0 \\
0 & 0 & \gamma & -d-\lambda
\end{array}\right|=0
$$

$$
\begin{gathered}
(-a-\lambda)\left|\begin{array}{ccc}
-b-\lambda & (-\alpha \Lambda) / a & 0 \\
\beta & -c-\lambda & 0 \\
0 & \gamma & -d-\lambda
\end{array}\right|=0 \\
(-a-\lambda)(-d-\lambda)\left|\begin{array}{cc}
-b-\lambda & (-\alpha \Lambda) / a \\
0 & -c-\lambda
\end{array}\right|=0 \\
(-a-\lambda)(-d-\lambda)(-b-\lambda)(-c-\lambda)=0
\end{gathered}
$$

Thus the four eigen values of the matrix are found as $\lambda_{1}=-a \lambda_{2}=-d \lambda_{3}=-b \lambda_{4}=-c$

$$
\begin{gathered}
\lambda_{1}=-(\delta+\mu) \lambda_{2}=-(\rho+\delta+\mu) \lambda_{3}=-(\beta+\delta+\mu) \lambda_{4} \\
=-(\gamma+\delta+\mu)
\end{gathered}
$$

Therefore, it is concluded that the LSDFEP $E_{0}$ of the system of differential equations (1)-(4) is locally asymptotically stable due to all eigen value is negative.

\subsection{Global Stability Analysis of Endemic Equilibrium Point}

The Global stability Analysis of endemic equilibrium point $E^{*}\left(S^{*}, E^{*}, I^{*}, R^{*}\right)$ is stated in Theorem 5 and proved by taking appropriate liapunove function. [7, 8]

Theorem 5 [Global Stability of endemic equilibrium point (GSEEP)] The endemic equilibrium point

$E^{*}\left(S^{*}, E^{*}, I^{*}, R^{*}\right)$ is globally asymptotically stable. Proof:

Let

$$
\begin{gathered}
L(S, \quad E, \quad I, \quad R)=\mathrm{m}_{1}\left(\mathrm{~s}-\mathrm{s}^{*}\right)^{2} / 2+\mathrm{m}_{2}\left(\mathrm{E}-\mathrm{E}^{*}\right)^{2} / 2+\mathrm{m}_{3}\left(\mathrm{I}-\mathrm{I}^{*}\right)^{2} / 2+\mathrm{m}_{4}\left(\mathrm{R}-\mathrm{R}^{*}\right)^{2} / 2[7,8] \\
d L / d t=\mathrm{m}_{1}\left(\mathrm{~s}-\mathrm{s}^{*}\right)\{\mathrm{ds} / \mathrm{dt}\}+\mathrm{m}_{2}\left(\mathrm{E}-\mathrm{E}^{*}\right)\{\mathrm{dE} / \mathrm{dt}\}+m_{3}\left(\mathrm{I}-\mathrm{I}^{*}\right)\{d I / d t\}+m_{4}\left(\mathrm{R}-\mathrm{R}^{*}\right)\{d R / d t\}
\end{gathered}
$$

Now substitute the model equation (1)-(4) into (15)

$$
\begin{aligned}
\mathrm{dL} / \mathrm{dt}=\mathrm{m}_{1}\left(\mathrm{~s}-\mathrm{s}^{*}\right)\{\Lambda & +\rho R-\alpha S I-a S\}+\mathrm{m}_{2}\left(\mathrm{E}-\mathrm{E}^{*}\right)\{\alpha S I-b E\} \\
& +\mathrm{m}_{3}\left(\mathrm{I}-\mathrm{I}^{*}\right)\{\beta E-c I\} \\
& +\mathrm{m}_{4}\left(\mathrm{R}-\mathrm{R}^{*}\right)\{\gamma I-d R\}
\end{aligned}
$$

Take out S, E, I, Rand put as change

$$
\begin{aligned}
\mathrm{dL} / \mathrm{dt}=\mathrm{m}_{1}\left(\mathrm{~s}-\mathrm{s}^{*}\right)\left(\mathrm{s}-\mathrm{s}^{*}\right)\{ & {[(\Lambda+\rho R) / S]-\alpha I-a\}+\mathrm{m}_{2}\left(\mathrm{E}-\mathrm{E}^{*}\right)\left(\left(\mathrm{E}-\mathrm{E}^{*}\right)\right)\{(\alpha S I / E)-b\} } \\
& +\mathrm{m}_{3}\left(\mathrm{I}-\mathrm{I}^{*}\right)\left(\mathrm{I}-\mathrm{I}^{*}\right)\{(\beta E / I)-c\} \\
+ & \mathrm{m}_{4}\left(\mathrm{R}-\mathrm{R}^{*}\right)\left(\mathrm{R}-\mathrm{R}^{*}\right)\{(\gamma I / R)-d\}
\end{aligned}
$$

By rearranging and take out negative sign from the bracket it could be obtained as

$$
\begin{aligned}
\mathrm{dL} / \mathrm{dt}=-\mathrm{m}_{1}\left(\mathrm{~s}-\mathrm{s}^{*}\right)^{2}\{- & {[(\Lambda+\rho R) / S]+\alpha I+a\}-\mathrm{m}_{2}\left(\mathrm{E}-\mathrm{E}^{*}\right)^{2}\{-(\alpha S I / E)+b\} } \\
& -\mathrm{m}_{3}\left(\mathrm{I}-\mathrm{I}^{*}\right)^{2}\{-(\beta E / I)+c\} \\
- & \mathrm{m}_{4}\left(\mathrm{R}-\mathrm{R}^{*}\right)^{2}\{-(\gamma I / R)+d\}
\end{aligned}
$$

Thus it is possible to set $m_{1}, m_{2}, m_{3}, m_{3}$ are non negative integers such that $\mathrm{dL} / \mathrm{dt} \leq 0$ and endemic equilibrium point 
is globally stable.

\section{Numerical Simulation}

In this section, the numerical simulation of model equations (1)-(4) is carried out using the software DE Discover 2.6.4. For Simulation purpose, a set of meaningful values are assigned to the model parameters. A set of initial conditions are given to the model variables. These sets of parametric values are given in Tables 4 and Model equations and parameter is arranged for DEDiscover software in this way for simulation purpose:

$\mathrm{dS} / \mathrm{dt}=$ Lambda + Rho $* \mathrm{R}-$ Alpha $* \mathrm{~S} * \mathrm{I}-($ Delta $+\mathrm{Mu}) * \mathrm{~S} / /$ the number of Susceptible class

$\mathrm{dE} / \mathrm{dt}=$ Alpha*S*I-(Beta + Delta $+\mathrm{Mu}) * \mathrm{E} / /$ the number of Exposed class

$\mathrm{dI} / \mathrm{dt}=$ Beta*E-(Gamma+Delta $+\mathrm{Mu}) * \mathrm{I} / /$ the number of infective class

$\mathrm{dR} / \mathrm{dt}=$ Gamma*I-(Rho+Delta $+\mathrm{Mu}) * \mathrm{R} / /$ the number of Recovered class

Table 4. Parameter values used for Simulation.

\begin{tabular}{lll}
\hline Parameter & Value & Reference \\
\hline$\Lambda$ & 271.230 & {$[3]$} \\
$\alpha$ & 2.50000 & {$[2]$} \\
$\beta$ & 0.14280 & {$[1]$} \\
$\gamma$ & 0.06600 & {$[3]$} \\
$\rho$ & 0.000095 & {$[5]$} \\
$\delta$ & 0.13100 & estimated \\
$\mu$ & 0.000001 & {$[5]$} \\
\hline
\end{tabular}

Using the parameter values given in Table 2 with different initial conditions in model equations (1)-(4) a simulation is done and the results are given in Figure 2-5.

From Figure 2, it is observed that when the number of exposed individual increases then the number of infected people will increase that leads to the decrease on the susceptible and recovered populations. Figure 3, shows all those class of population changing for some time and eventually the populations will be constant. Thus in any situations there will exist those class of populations and continue in stable state after some time in future.

From Figure 4, recovered and infected populations are inversely proportional. That is if the population gets greater the number of infection, then will be less the number of recovery may be due to lack of resources or management, and Similarly, from Figure 5, exposed and infected populations are directly proportional. If there is more infection in the community, there will be more exposed individual and the less infected community, the less the exposed community.

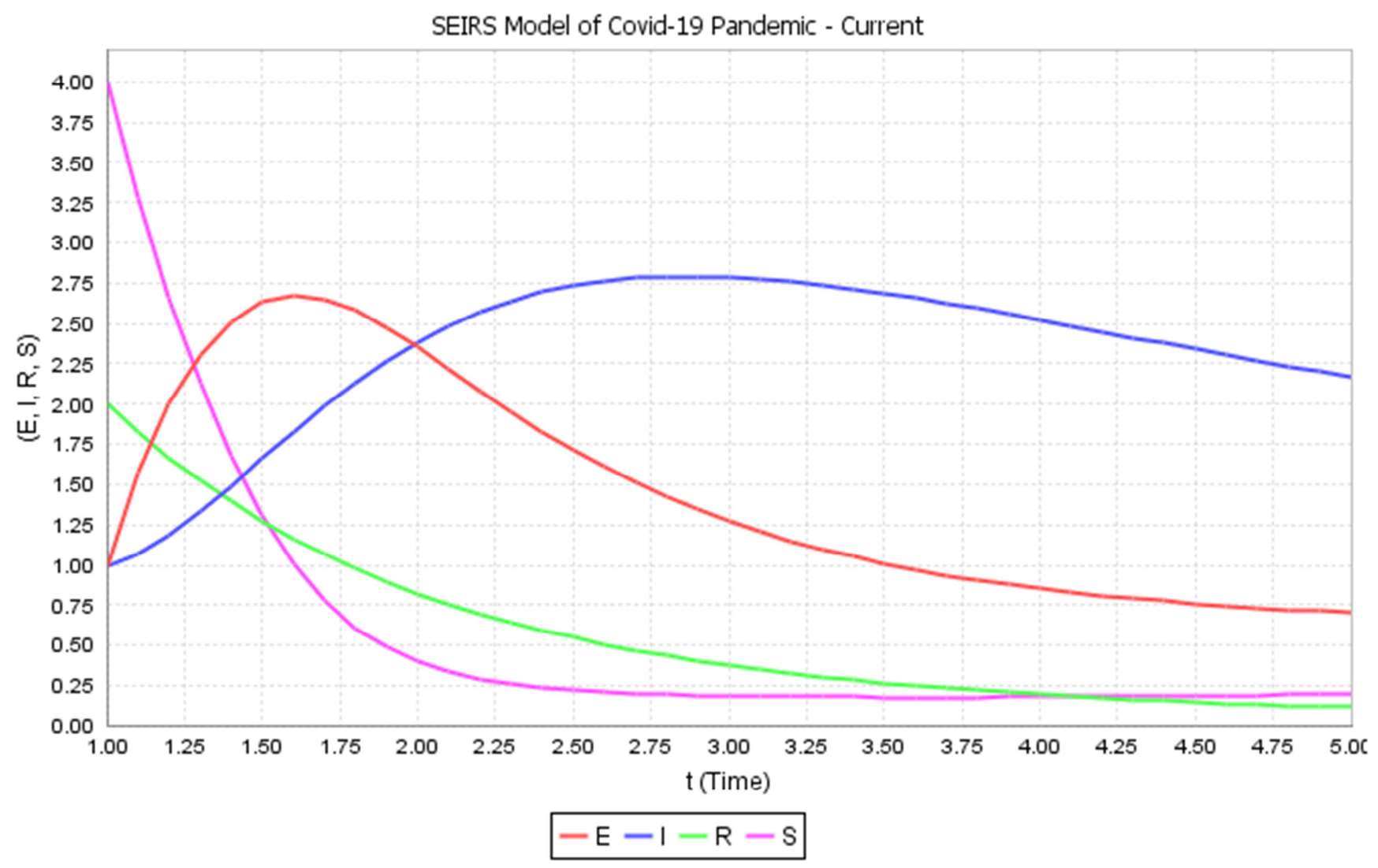

Figure 2. Time series plot for all variables with different initial condition. 


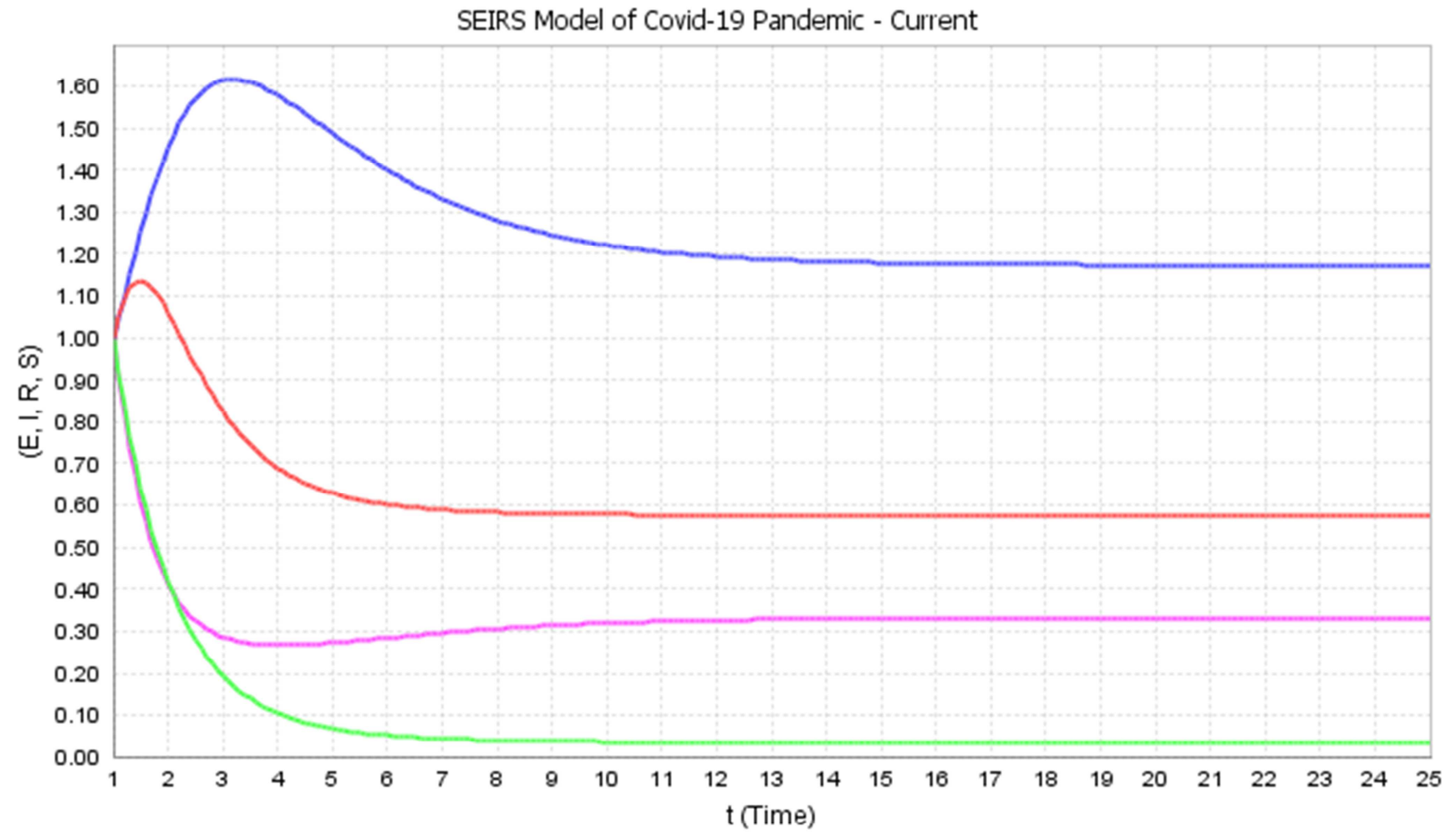

$$
-\mathrm{E}-\mathrm{I}-\mathrm{R}-\mathrm{S}
$$

Figure 3. Time series plot for all variables with same initial condition.

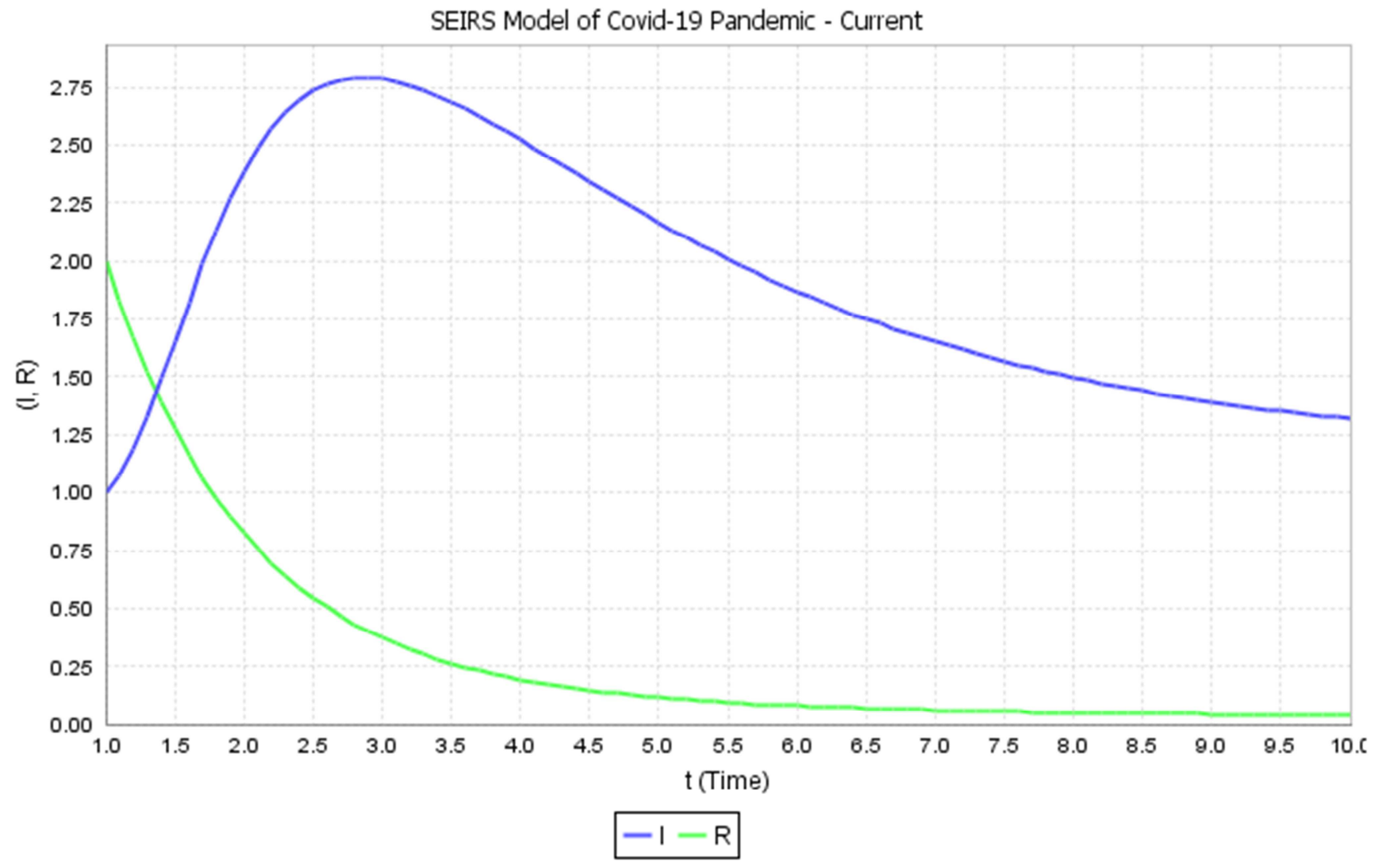

Figure 4. Time series plot for infected and recovered population. 


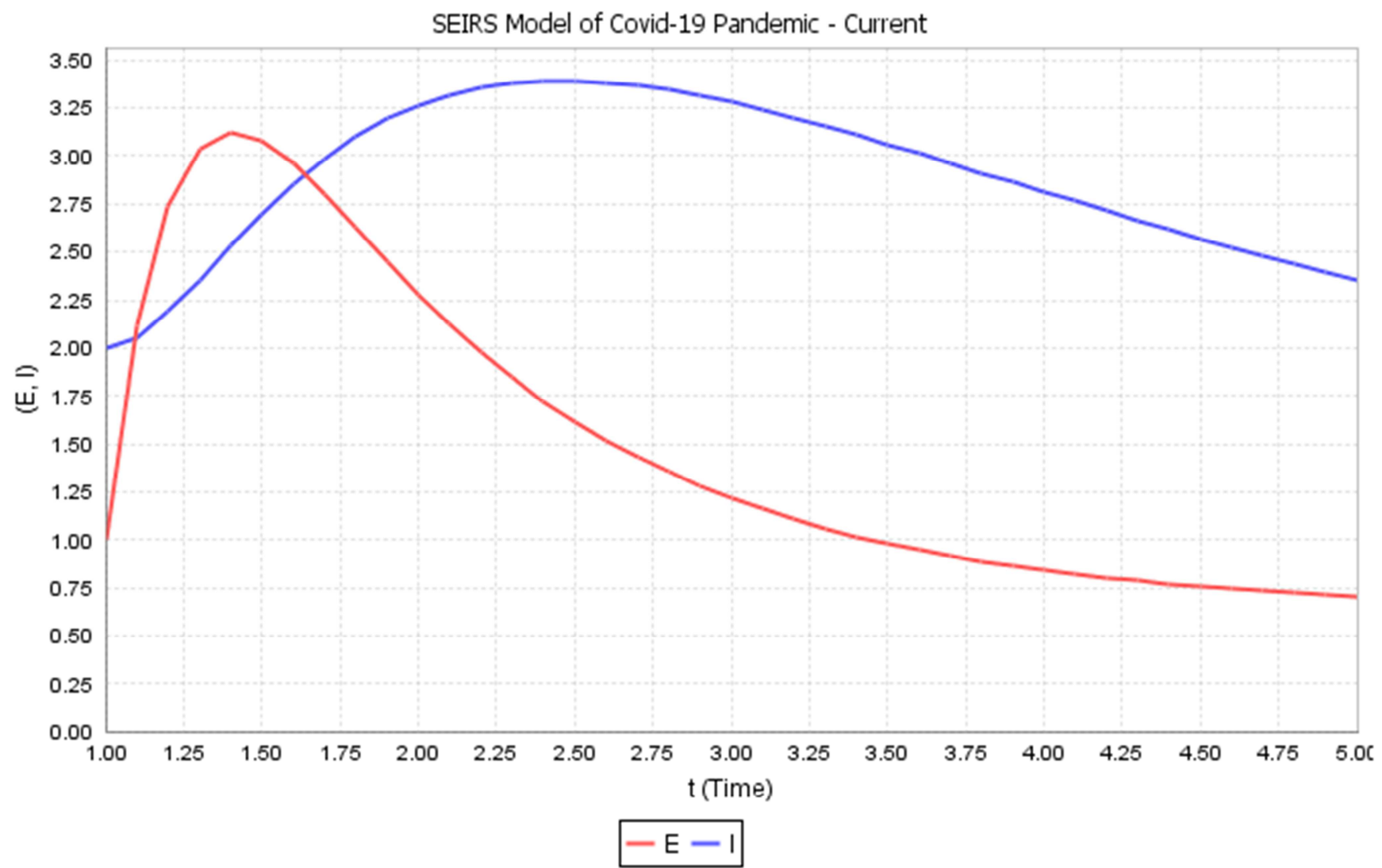

Figure 5. Time series plot for exposed and infected population.

\section{Sensitivity Analysis}

Sensitivity analysis tells us how important each parameter is to disease transmission. It is used to discover parameters that have a high impact on $R_{0}$ and should be targeted by intervention strategies. Sensitivity indices allow us to measure the relative change in a variable when a parameter changes. The normalized forward sensitivity index of a variable with respect to a parameter is the ratio of the relative change in the variable to the relative change in the parameter. If the result is negative, then the relationship between the parameters and $R_{0}$ is inversely proportional. In this case, we will take the modulus of the sensitivity index so that we can deduce the size of the effect of changing that parameter. On the other hand, a positive sensitivity index means an increase in the value of a parameter.

A highly sensitive parameter should be carefully estimated, because a small variation in that parameter will lead to large quantitative changes. An insensitive parameter, on the other hand, does not require as much effort to estimate, since a small variation in that parameter will not produce large changes to the quantity of interest $[9,11,13]$

The explicit expression of $R_{0}$ is given by

$R_{0}=[\alpha \beta \Lambda] /[(\delta+\mu)(\beta+\delta+\mu)(\gamma+\delta+\mu)]$. Since $R_{0}$ depends only on six parameters, we derive an analytical expression for its sensitivity to each parameter using the normalized forward sensitivity index as follows:
Table 5. Sensitivity of $R_{0}$ for the Parameter values.

\begin{tabular}{lll}
\hline Parameter & Sensitivity index of $\boldsymbol{R}_{\mathbf{0}}$ & Sign \\
\hline$\Lambda$ & $Y_{\Lambda}^{R_{0}}=\frac{\partial R_{0}}{\partial \Lambda} \times \frac{\Lambda}{R_{0}}=1$ & $+\mathrm{ve}$ \\
$\alpha$ & $Y_{\alpha}^{R_{0}}=\frac{\partial R_{0}}{\partial \alpha} \times \frac{\alpha}{R_{0}}=1$ & $+\mathrm{ve}$ \\
$\beta$ & $Y_{\beta}^{R_{0}}=\frac{\partial R_{0}}{\partial \beta} \times \frac{\beta}{R_{0}}=\frac{\delta \mu}{(\beta+\delta \mu)^{2}}$ & $+\mathrm{ve}$ \\
$\gamma$ & $Y_{\gamma}^{R_{0}}=\frac{\partial R_{0}}{\partial \gamma} \times \frac{\gamma}{R_{0}}=\frac{-\gamma}{\gamma+\delta+\mu}$ & $-\mathrm{ve}$ \\
$\delta$ & $Y_{\delta}^{R_{0}}=\frac{\partial R_{0}}{\partial \alpha} \times \frac{\delta}{R_{0}}=\frac{-\delta[(a+b) c+a b]}{a b c}$ & $-\mathrm{ve}$ \\
$\mu$ & $Y_{\mu}^{R_{0}}=\frac{\partial R_{0}}{\partial \alpha} \times \frac{\mu}{R_{0}}=\frac{-\mu[(a+b) c+a b]}{a b c}$ & $-\mathrm{ve}$ \\
\hline
\end{tabular}

where, $\quad a=\delta+\mu, b=\beta+\delta+\mu, c=\gamma+\delta+\mu, d=\rho+$ $\delta+\mu$, and From the Table 5 , It is to be noted that the parameters $\Lambda, \alpha, \beta$ are positive and hence play a vital role in controlling the stability aspects of the system, and the remaining parameters are negative and hence have not big influence on the system.

\section{Result and Discussion}

A mathematical SEIRS model of COVID-19 was Conducted and the sensitivity indices of the basic reproduction number $R_{0}$ is Computed to determine the relative importance of the model parameters in the disease transmission. This information leads us to identify the influence of each model parameter in the basic reproduction number. Consequently, it is helpful to know 
and predict the disease progress, persistent or decline in the past, present and future.

This mathematical model analysis may provide critical information for decision makers and public health officials, who may have to deal with infectious disease of COVID-19 pandemic. I hope that the paper will have a great impact on citizens of the whole world to concentrate on prevention and control of COVID-19 pandemic. Such contribution is interesting regarding to COVID-19, which causes a large disruption in the lives of sufferers and has enormous socioeconomic costs occurred in the world.

Almost all latest research recommend the following protective measures: hygiene, masks, physical distancing, staying indoors, boosting immune system, raising awareness through networking (like face book, and twitter), boosting moral of front-line workers such as (medical practitioners, nursing staff, cleaning staff, health care centers) who are interacting directly with the patients.

\section{Conclusion}

In this Paper, SEIRS mathematical model describing the dynamics of COVID-19 is formulated and analyzed. The model is developed based on biologically reasonable assumptions. The mathematical analysis has shown that if basic reproduction number is less than one, then number of cases decrease over time and eventually the disease die out, and if the basic reproduction number is equals to one, then cases are stable. On the other hand, if the basic reproduction number is greater than one then the number of cases increase over time gets worth, and the disease continue to spread more rapidly.

Moreover, existence, positivity and boundedness of the solution of the model is shown to clarify the model is biologically meaningful and mathematically well posed. Stability analysis of the model is checked with help of next generation matrix and global stability are proved by taking appropriate liapunove function.

\section{Acknowledgements}

The authors would like to thank the editor and the anonymous reviewers of the journal AJAM for their helpful suggestions and remarks.

\section{References}

[1] Fang Y, Nie Y, Penny M (2020). Transmission dynamics of the COVID-19 outbreak and effectiveness of government interventions: A data-driven analysis. J Med Virol, 1-15. https://doi.org/10.1002/jmv.25750.
[2] Yang C, Wang J (2020). A mathematical model for the novel corona virus epidemic in Wuhan, China. Mathematical biosciences and engineering 17 (3), 2708-2724.

[3] Okhuese (2020) A, mathematical predictions for covid-19 as a global pandemic. Research Gate.

[4] Bhola J, Revathi V, and Koul M. Corona Epidemic in Indian context: Predictive Mathematical Modeling, medRxiv preprint doi: https://doi.org/10.1101/2020.04.03.20047175.

[5] Binti Hamzah FA, Lau C, Nazri H, Ligot DV, Lee G, Tan CL, (19 March 2020) Corona Tracker: World-wide COVID-19 Outbreak Data Analysis and Prediction. Bull World Health Organ. doi: http://dx.doi.org/10.2471/BLT.20.255695.

[6] P. Zhou, X. L. Yang, X. G. Wang, B. Hu, L. Zhang, W. Zhang, (2020). Discovery of a novel corona virus associated with the recent pneumonia outbreak in humans and its potential bat origin, bioRxiv.

[7] Abayneh Fentie Bezabih, Geremew Kenassa Edessa, Koya Purnachandra Rao (2020) Mathematical Eco-Epidemic Model on Prey-Predator System. IOSR Journal of Mathematics (IOSR-JM), 16 (1), pp. 22-34.

[8] Alfred Hugo A, Estomih S. Massawe, and Oluwole Daniel Makinde (July 2012). An Eco-Epidemiological Mathematical Model with Treatment and Disease Infection in both Prey and Predator Population. Journal of Ecology and natural environment Vol. 4 (10), pp. 266-273,.

[9] Tadele Tesfa Tegegne, Purnachandra Rao Koya, and Temesgen Tibebu Mekonnen (2016) Impact of Heterosexuality \& Homosexuality on the transmission and dynamics of HIV/AIDS, IOSR Journal of Mathematics (IOSR-JM), Volume 12,Issue 6 Ver, PP 38-49.

[10] P. van den Driessche and James Watmough (2002). Reproduction numbers and sub-threshold endemic equilibria for compartmental models of disease transmission. Mathematical Biosciences 180 (2002), 29-48.

[11] Selam Nigusie Mitku, Purnachandra Rao Koya (2017). Mathematical Modeling and Simulation Study for the Control and Transmission Dynamics of Measles. American Journal of Applied Mathematics. Vol. 5, No. 4, pp. 99-107. doi: 10.11648/j.ajam.20170504.

[12] Sachin Kumar and Harsha Kharbanda, (29 sep 2017). Stability Analysis of Prey-Predator Model With Infection, Migration and Vaccination In Prey, arXiv: 1709.10319vl [math. DS]

[13] Helena Sofia Rodrigues, M. Teresa T. Monteiro, and Delfim F. M. Torres, (2013). Sensitivity Analysis in a Dengue Epidemiological Model. Hindawi Publishing Corporation Conference Papers in Mathematics. Volume 2013, Article ID 721406, 7 pages. 